\title{
Preface to the special issue on "Concept Lattice and their Applications" (CLA-2011)
}

\author{
Amedeo Napoli
}

Published online: 16 November 2013

(C) Springer Science+Business Media Dordrecht 2013

This special issue contains the extended versions of selected papers that were presented at CLA-2011, the Eighth International Conference on "Concept Lattices and their Applications". CLA-2011 was held in Nancy, France on October 17-20, 2011, and was organized by LORIA/Inria Nancy Grand Est and the University of Nancy (http://cla2011.loria.fr/). CLA is one of the few international conferences dedicated to Formal Concept Analysis (FCA) and related applications (http://cla.inf.upol.cz/). The areas of interest of CLA include foundations of FCA, applications of FCA, and related topics such as data mining, knowledge discovery, data and knowledge engineering, and data analysis. Foundations are in concern with posets, lattice theory, logic, concept lattices, attribute implications, association rules, data dependencies (e.g. functional dependencies) and FCA algorithms. Applications are concerned with visualization, data preprocessing, dimensionality reduction, classification and clustering, information retrieval and ontology engineering.

The program of CLA-2013 included a special afternoon session on "Galois Connections and Residuation: Origins and Developments" and invited presentations on "Metrics, Betweeness Relations, and Entropies on Lattices", "Mathematical Morphology and Lattices", "Canonical Extensions and Duality Theory" and "Random Concept Lattices". This shows the extreme diversity of the research on concept lattices and the wide range of possible applications.

Following tradition, a special journal issue was organized for collecting and publishing extended newly refereed versions of the best papers of CLA-2013. It is our pleasure that Professor Golumbic, the Editor-in-Chief of the "Annals of Mathematics and Artificial Intelligence", accepted our proposal to organize this special issue. Each submitted paper was reviewed by three or four reviewers who are experts in the field of FCA and related areas. In addition, and according to the journal policy, the papers co-authored by the guest editor were directly handled by the Editor-in-Chief. Seven papers were finally selected for this special issue, that we are pleased to present hereafter.

\footnotetext{
A. Napoli $(\varangle)$

LORIA (CNRS - INRIA Nancy Grand Est - Université de Lorraine)

BP 239 - 54506 Vandoeuvre-les-Nancy, France

e-mail: Amedeo.Napoli@loria.fr
} 
In their paper entitled "Optimizations in Computing the Duquenne-Guigues Basis of Implications", Konstantin Bazhanov and Sergei Obiedkov consider algorithms involved in the computation of the Duquenne-Guigues basis of implications. They show that the most widely used algorithm for constructing the basis, which is Ganter's Next Closure, can be optimized and they proposed such an optimization. Then they compare the performance of the original algorithm and its optimized version in a series of experiments using artificially generated and real-life data sets. The Duquenne-Guigues basis is one of the most important and useful implication bases in FCA.

The paper entitled "Fast Algorithms for Implication Bases and Attribute Exploration Using Proper Premises" by Uwe Ryssel, Felix Distel and Daniel Borchmann, is also related to implication bases. Here, the purpose is to present a new algorithm for the fast computation of the so-called "proper premises" as an alternative to the Duquenne-Guigues basis. The resulting implication basis is not minimal in terms of cardinality but is less costly in terms of runtime. The algorithm is based on a link between proper premises and minimal hypergraph transversals, and shows a very good computational behavior.

"Biclustering Meets Triadic Concept Analysis" is the title of the paper by Mehdi Kaytoue, Sergei O. Kuznetsov, Juraj Macko and Amedeo Napoli. Biclustering numerical data is a popular data-mining task aimed at several applications, e.g., gene expression data analysis and recommendation. A bicluster reflects a strong association between a subset of objects and a subset of attributes in a numerical object/attribute data-table. The authors introduce new and important links between biclustering and FCA through Triadic Concept Analysis, an extension of FCA to ternary relations. They propose a formal framework for biclustering and a method for enumerating numerical biclusters which is complete, correct and non-redundant. This paper provides a nice and efficient application of Triadic Concept Analysis.

Laszlo Szathmary, Petko Valtchev, Amedeo Napoli, Robert Godin, Alix Boc and Vladimir Makarenkov are interested in pattern mining and association rule mining in their paper entitled "A Fast Compound Algorithm for Mining Generators, Closed Itemsets, and Computing Links Between Equivalence Classes". This research work is based on an indepth analysis of the interplay between the mining of frequent closed itemsets (FCIs), the mining of frequent generators (FGs), and the computation of the precedence relation between FCIs, interplay which is rooted in a fundamental duality from hypergraph theory. The authors propose a new algorithmic schema which enables a more parsimonious computation, a very good efficiency, and capabilities to focus on significant associations.

In their paper "Recursive Decomposition Tree of a Moore Co-Family and Closure Algorithm", Pierre Colomb, Alexis Irlande, Olivier Raynaud and Yoan Renaud are interested in Moore families and co-families. The former are collections of sets closed under intersection and contain the base set, while the later are closed under union and contain the empty set. The authors introduce a recursive decomposition tree of any Moore co-family and propose an original algorithm for computing the closure under union of any family.

The two last papers are more related to applications of FCA. Ingrid Falk and Claire Gardent, in their paper entitled "Combining Formal Concept Analysis and Translation to Assign Frames and Semantic Role Sets to French Verbs", propose a novel approach for designing verb classifications based on stability and separation indices which allow to extract verb classes of interest from a concept lattice (built from existing linguistic resources from French). The approach is very interesting as it supports polysemy and provides a syntactic and semantic characterization of the resulting verb classes. This is one nice application of FCA to natural language processing. 
In the last paper, "Boolean Factors as a Means of Clustering of Interestingness Measures of Association Rules", Radim Belohlavek, Dhouha Grissa, Sylvie Guillaume, Engelbert Mephu Nguifo and Jan Outrata explore Boolean factor analysis for classifying interest measures used in association rule mining. The resulting clustering of the measures reveals overlapping clusters for which the authors provide interpretations. This clustering is compared to previous approaches and open new possibilities in the interpretation of interest measures and their usefulness in association rule mining.

Finally, we would like to express our thanks to the authors who submitted extended versions of their papers to this special issue as well as to the reviewers for their diligent and valuable work. We hope that this special issue will be a useful and an actual source of information on concept lattices and their applications. 